\title{
Antiplatelet Therapy prior to Temporary Stent-Assisted Coiling
}

W e read with interest the article by Signorelli et $\mathrm{al}^{1}$ describing their experience with 8 patients treated with a Solitaire stent (Covidien, Irvine, California) deployed temporarily to enable stent-assisted coiling of cerebral aneurysms. This article expands on the technique that we described earlier. ${ }^{2}$ Unlike in our article, Signorelli et al performed their procedures without preadministering antiplatelet therapy. We would like to caution against the wide adoption of this technique without pretreatment with antiplatelet agents.

Since the publication of our article, ${ }^{2}$ we have treated 10 aneurysms in 8 patients by using this technique, raising our experience to 13 procedures. This technique was used for the treatment of wide-neck aneurysms of the anterior communicating artery $(n=$ $4)$, internal carotid artery termination $(n=2)$, basilar artery tip $(n=2)$, middle cerebral artery bifurcation $(n=1)$, or the posterior communicating artery $(n=1)$.

The technique was performed after antiplatelets were started in all except 4 cases. In addition, we used it to treat 6 unruptured aneurysms, a unique feature of our experience compared with the literature on this technique. One of the patients with an unruptured aneurysm was not pretreated with dual antiplatelet therapy because the aneurysm was thought to be amenable to primary or, if needed, balloon-assisted coiling. However, a stent was required because the neck of the aneurysm was wider than anticipated, and temporary stent coiling was planned. The procedure was complicated by in-stent thrombosis requiring intravenous abciximab,

http://dx.doi.org/10.3174/ajnr.A4090 and no clinical sequelae were noted after the procedure. This experience highlights the thromboembolic risks that can be associated with this technique, especially when it is used without prior antiplatelet therapy. To better appreciate the extent of these risks, a large case series reporting on this technique is required.

We believe that this technique avoids many of the potential complications that can accompany balloon-assisted coiling and mitigates the need for life-long antiplatelet therapy as in the standard stent-assisted coiling. Therefore, we recommend considering this approach, with experienced operators, in all candidates with ruptured, and selectively in unruptured, aneurysms.

\section{REFERENCES}

1. Signorelli F, Gory B, Turjman F. Temporary Solitaire stent-assisted coiling: a technique for the treatment of acutely ruptured wideneck intracranial aneurysms. AJNR Am J Neuroradiol 2014;35: 984-88

2. Almekhlafi MA, Hockley A, Wong JH, et al. Temporary Solitaire stent neck remodeling in the coiling of ruptured aneurysms. J Neurointerv Surg 2013;5(suppl 3):iii76-78

M.A. Almekhlafi

Departments of Radiology and Clinical Neurosciences University of Calgary

Calgary, Alberta, Canada Faculty of Medicine King Abdulaziz University Jeddah, Saudi Arabia M. Goyal

Departments of Radiology and Clinical Neurosciences University of Calgary Calgary, Alberta, Canada 